\title{
A Semi-Distributed Approach for Uplink Max-Min Energy Efficiency Optimization with Minimum User Satisfaction and Adjacency Constraints
}

\author{
Iran M. Braga Jr., F. Rafael M. Lima, Tarcisio F. Maciel and Victor F. Monteiro
}

\begin{abstract}
Maximizing energy efficiency is one of the pillars of modern networks. In this context, we consider in this letter a nonlinear max-min energy efficiency problem in the uplink of wireless networks. Due to the problem nonlinearity we resort to epigraph form so as to obtain an integer linear problem and to propose a centralized optimal solution for it using a branch-andbound algorithm. Also because distributed solutions are useful to deal with high computational processing and scalability problems, we propose a low-complexity semi-distributed solution for the problem using a specific signaling scheme. Simulations show that the proposed semi-distributed solution performs closely to the centralized optimal scheme and outperforms state-of-the-art algorithms in terms of energy efficiency and outage rate.
\end{abstract}

Index Terms-Energy Efficiency, SC-FDMA, QoS.

\section{INTRODUCTION}

Single carrier - frequency division multiple access (SCFDMA) has been adopted in the uplink of Long Term Evolution (LTE) systems motivated by its attractive peak-to-average power ratio (PAPR) characteristics. SC-FDMA often requires that the resource blocks assigned on uplink should be adjacent to each other in the frequency domain. Allocation of contiguous resource blocks, is also specified in the 5 th generation $(5 \mathrm{G})$ new radio (NR) in [1]. However, as shown in [2], the adjacency constraints of SC-FDMA is sufficient to turn radio resource allocation (RRA) problems non-polynomial time (NP)-hard.

In the last years, some works have focused on RRA solutions to SC-FDMA networks. In [3] three resource and user scheduling algorithms with contiguous frequency resource allocation were proposed. However, the authors focused on modified largest weighted delay first as scheduling criterion. Driven by the increase in energy consumption in wireless networks, studies on energy efficiency (EE) have been considered in [4] [7]. In [4] the sum-power minimization problem was studied as an integer binary optimization problem, and a low-complexity solution was proposed by assuming canonical duality theory. In [5] the problem of minimizing the energy consumption per transmitted bit in uplink SC-FDMA was proposed. In [6] the authors proposed a maximum-power decrease-first (MPDF) greedy algorithm based on round robin for minimizing the energy consumption per transmit bit. Finally, in [7] the optimal solution and a heuristic suboptimal energy-efficient (HSO-EE) algorithm with low computational complexity were proposed to maximize the sum of individual EE.

This work was supported in part by Ericsson Research, Technical Cooperation contracts UFC.48 and UFC.49 and in part by the Coordenação de Aperfeiçoamento de Pessoal de Nível Superior - Brasil (CAPES) - Finance Code 001, in part by CAPES/PRINT Grant $88887.311965 / 2018-00$, in part by FUNCAP under Grant BP4-0172-00245.01.00/20 and in part by CNPq under Grant 308621/2018-2.

The authors are with the Wireless Telecom Research Group (GTEL), Federal University of Ceará, Fortaleza 60455-760, Brazil. E-mails: \{iran, rafaelm, maciel, victor\}@gtel.ufc.br.

Digital Object Identifier: 10.14209/jcis.2021.9
Although these works have considered the adjacency constraint as well as quality of service (QoS) aspects, the multiservice scenario was ignored. In addition, the authors also ignored the per-service minimum satisfaction guarantees. We define per-service satisfaction as a minimum percentage (or number) of user equipments (UEs) of each service that should have their QoS fulfilled. The per-service satisfaction concept was considered in [8] and [9]. In [8] the constrained power minimization (CPM) problem was considered to minimize the total power consumption while in [9] the constrained energy efficiency maximization (CEEM) problem was studied in order to maximize the overall EE. However, works [8] and [9] only considered centralized solutions. Indeed, centralized solutions require global channel state information (CSI) availability at a central controlling unit, which may be difficult to acquire in modern networks due to the amount of involved network nodes. A distributed approach was proposed in [10], but the per-service satisfaction was ignored.

In this work, we study the max-min EE problem subject to minimum user satisfaction and adjacency constraints in the uplink of wireless networks. In summary, the main contributions of this letter are: (1) Formulation and modeling of a new EE optimization problem with minimum user satisfaction and adjacency constraints; (2) Proposal of a centralized optimal solution using a branch-and-bound (BB) algorithm; (3) Proposal of a semi-distributed low-complexity solution with a signaling scheme for practical system deployments; (4) Characterization of the performance/complexity trade-off of the involved algorithms, and; (5) Performance evaluation by means of simulations of the proposed solution compared to state-of-the-art algorithms.

\section{SYSTEM MODEL}

We assume the uplink of a cellular system with $U$ singleantenna UEs connected to a single-antenna evolved node B (eNB). We consider that there is a total of $K$ available resource blocks (RBs) and we define $\mathcal{U}$ and $\mathcal{K}$ as the sets of UEs and RBs, respectively. In addition, we assume a multiservice scenario in which a total of $S$ services are provided with $\mathcal{S}$ being the set of all services. Each service $s \in \mathcal{S}$ requires a minimum number $\left(\psi_{s}\right)$ of UEs that should be satisfied. We also define $\mathcal{U}_{s}$ as the set of UEs that are associated to service $s$ and the cardinality of set $\mathcal{U}_{s}$ is $U_{s}$. Note that each UE subscribes to only a single service. Moreover, $\tau_{u}$ consists of a QoS requirement for each UE $u$ in terms of throughput.

Due to the adjacency constraint imposed by SC-FDMA, the number of resource assignment patterns that can be build with $K$ RBs is given by $N=K^{2} / 2+K / 2+1$ [8]. We define $\mathcal{N}$ as the set of all possible resource assignment patterns. Besides that, we define the matrix $\mathbf{A}$ whose element $a_{k, n}$ assumes the value 1 if the RB $k \in \mathcal{K}$ belongs to the assignment pattern $n \in \mathcal{N}$, and 0 otherwise. 
We assume $M$ modulation and coding scheme (MCS) levels contained in set $\mathcal{M}$, where a given UE transmits on MCS level $m \in \mathcal{M}$ when its effective signal to noise ratio (SNR) is within the interval $\left[\gamma_{m}, \gamma_{m+1}\right)$, with $\gamma_{m}<\gamma_{m+1}$. The transmission with MCS level $m$ leads to a transmit data rate equal to $r_{m}$. Note that SC-FDMA also imposes two other constraints in order to assure low PAPR: the same MCS and the same transmit power level should be employed in all RBs assigned to a given UE. In addition, we employ the Minimum Mean Square Error (MMSE) equalizer to combat Inter Symbol Interference (ISI). Thus, the effective SNR perceived by the receiver when data is transmitted by UE $u$ on the RBs that belong to assignment pattern $n, \gamma_{u, n}^{\mathrm{MMSE}}$, is [8], [9]

$$
\gamma_{u, n}^{\mathrm{MMSE}}=\left(\left(\frac{1}{c \cdot K_{n}} \sum_{k=1}^{K_{n}} \sum_{z=1}^{c} \frac{\gamma_{u, z, k}}{\gamma_{u, z, k}+1}\right)^{-1}-1\right)^{-1},
$$

where $c$ is the number of subcarriers per RB, $K_{n}$ is the number of RBs in the assignment pattern $n$ and $\gamma_{u, z, k}$ is the SNR experienced in the link between the UE $u$ and eNB when transmitting on the $z$-th subcarrier of RB $k$ given by

$$
\gamma_{u, z, k}=\left(\left(p_{u} /\left(c \cdot K_{n}\right)\right) \cdot g_{u, z, k}\right) / \sigma^{2},
$$

with $p_{u}$ being the transmit power of UE $u, \sigma^{2}$ is the noise power in the bandwidth of a subcarrier and $g_{u, z, k}$ is the total channel gain in the link between the UE $u$ and eNB on the $z$-th subcarrier of RB $k$. Note that the channel coefficients are kept approximately constant during a transmission time interval and we assume perfect CSI at eNB and UEs.

Let $f(\cdot)$ be the function mapping SNR to discrete data rate values. The transmit data rate when UE $u$ transmits on the RBs that belong to the assignment pattern $n$ with MCS $m$ is given by $r_{u, n, m}=f\left(\gamma_{u, n}^{\mathrm{MMSE}}\right)$. Then, to find the power required by UE $u$ using the assignment pattern $n$ to achieve the MCS level $m, p_{u, n, m}$, we should replace (2) in (1) and solve the equation with $\gamma_{u, n}^{\mathrm{MMSE}}=\gamma_{m}$.

\section{Problem Formulation}

In this letter, we focus on a max-min EE problem subject to adjacency and minimum user satisfaction constraints in the uplink of mobile networks. Let $\xi_{u, n, m}$ be the EE of UE $u$ using the assignment pattern $n$ in MCS level $m$ given by $\xi_{u, n, m}=r_{u, n, m} /\left(p_{u, n, m}+\varsigma\right)$, where $\varsigma$ is the circuit power. So, we formulate the problem as

$$
\begin{array}{rlrl}
\underset{x_{u, n, m}, \rho_{u}}{\operatorname{maximime}}\left\{\min _{\forall u}\left\{\sum_{n=1}^{N} \sum_{m=1}^{M} \xi_{u, n, m} \cdot x_{u, n, m}\right\}\right\}, & \\
\text { subject to } & \sum_{u=1}^{U} \sum_{n=1}^{N} \sum_{m=1}^{M} a_{k, n} \cdot x_{u, n, m} \leq 1, & & \\
& \sum_{n=1}^{N} \sum_{m=1}^{M} x_{u, n, m}=1, & \forall u \in \mathcal{K}, \\
& \sum_{n=1}^{N} \sum_{m=1}^{M} p_{u, n, m} \cdot x_{u, n, m} \leq P_{u}^{\max }, & \forall u \in \mathcal{U}, \\
& \sum_{n=1}^{N} \sum_{m=1}^{M} r_{u, n, m} \cdot x_{u, n, m} \geq \rho_{u} \tau_{u}, & \forall u \in \mathcal{U},
\end{array}
$$

$\forall u \in \mathcal{U}, \quad(3 \mathrm{c})$

$$
\begin{array}{lr}
\sum_{u=1}^{U_{s}} \rho_{u} \geq \psi_{s}, & \forall s \in \mathcal{S}, \\
\rho_{u} \in\{0,1\}, & \forall u \in \mathcal{U}, \\
x_{u, n, m} \in\{0,1\}, \forall u \in \mathcal{U}, n \in \mathcal{N} \text { and } m \in \mathcal{M},
\end{array}
$$$$
\forall u \in \mathcal{U},(3 \mathrm{~g})
$$

where $x_{u, n, m}$ is a binary optimization variable that assumes 1 if UE $u$ is assigned to the pattern $n$ and achieves the MCS level $m$ and 0 otherwise, $\rho_{u}$ is a selection variable that assumes 1 if UE $u$ is selected to be satisfied and 0 otherwise. The objective function shown in (3a) consists in maximizing the minimum EE. Constraint ( $3 \mathrm{~b}$ ) assures that the RBs cannot be shared among UEs of the same cell at the same time, while (3c) ensures that only one assignment pattern can be chosen by each UE. Constraint (3d) states that the power allocated by each UE cannot overcome the maximum power available, $P_{u}^{\max }$. Finally, the constraints (3e) and (3f) guarantee that a minimum number of UEs should have their QoS requirements fulfilled for each service.

\section{Centralized Optimal Solution}

Problem (3) belongs to the class of non-linear combinatorial optimization problems and can be transformed into an Integer Linear Problem (ILP) by using its epigraph form as follows

$$
\begin{aligned}
& \underset{x_{u, n, m}, \rho_{u}, \phi}{\operatorname{maximize}}\{\phi\}, \\
& \text { subject to (3b) to (3h) } \\
& \sum_{n=1}^{N} \sum_{m=1}^{M} \xi_{u, n, m} \cdot x_{u, n, m} \geq \phi, \quad \forall u \in \mathcal{U},
\end{aligned}
$$

where $\phi>0$ denotes the minimum EE of UEs. Now, the optimal solution of this problem can be obtained by standard methods such as the BB algorithm [11].

\section{Proposed Semi-Distributed Solution}

The centralized solutions such as the one presented in Section IV are in general very complex in terms of computational processing and signaling. Specifically, all problem inputs such as channel states per RB, should be made available at the eNB in order to solve problem (4). Therefore, distributed solutions arise as promising methods to deal with this issue.

That said, we propose a semi-distributed solution in which each UE reports to the eNB which assignment patterns fulfill both its power and QoS constraints. In other words, each UE $u$ is able to locally compute $r_{u, n, m}$ and $p_{u, n, m}$ for all $n \in \mathcal{N}$ and $m \in \mathcal{M}$ and, for each assignment pattern, it verifies which MCS levels fulfills both power and QoS constraints and selects only the MCS level with highest EE. Note that, if no MCS level fulfill the constraints, the UE can discard this assignment pattern. Thus, we define $\mathcal{N}_{u}^{*}$ as the set of feasible assignment patterns for UE $u$. Finally, the UE reports to the eNB the feasible assignment patterns and its respective MCS level.

After receiving the reports from UEs, the eNB can check which UEs have at least one feasible assignment pattern and define a new set, $\mathcal{U}^{*}$, with only the feasible UEs. Observe that, if the minimum number of satisfied UEs for a given service cannot be fulfilled (constraint (3e)), problem (4) is 
infeasible and an outage occurs ${ }^{1}$. On the other hand, if the minimum number of UEs satisfied per service is fulfilled, we can reformulate problem (4) as

$$
\begin{array}{cc}
\text { subject to } & \sum_{\substack{\max _{u, n} \\
\text { maxime }}} \sum_{n \in \mathcal{N}_{u}^{*}} a_{k, n} \cdot y_{u, n} \leq 1, \quad \forall k \in \mathcal{K} \\
\sum_{n \in \mathcal{N}_{u}^{*}} y_{u, n}=1, & \forall u \in \mathcal{U}^{*} \\
\sum_{n \in \mathcal{N}_{u}^{*}} \xi_{u, n} \cdot y_{u, n} \geq \phi, & \forall u \in \mathcal{U}^{*}, \\
y_{u, n} \in\{0,1\}, & \forall\left(u \in \mathcal{U}^{*}, n \in \mathcal{N}_{u}^{*}\right),
\end{array}
$$

where $\xi_{u, n}$ is the EE of UE $u$ using the assignment pattern $n$ and $y_{u, s}$ is a binary optimization variable that assumes 1 if the assignment pattern $n$ is allocated to UE $u$ and 0 otherwise.

Note that problem (5) is also an ILP, which can be solved optimally by the BB algorithm. Indeed, it is easy to see that the solution of problem (5) is the same of problem (4). However, the search space is reduced compared to problem (4) since this approach transfers to UEs part of the optimization tasks usually performed at the eNB side. Even so, the optimal solution for problem (5) is obtained at the cost of high computational complexity. Thus, we propose a low-complexity solution. The complete algorithm can be seen in Alg. 1 and is described in details below.

We start, in line 1 , by defining the auxiliary sets $\mathcal{U}^{+}$and $\mathcal{N}^{\dagger}$ equal to $\mathcal{U}^{*}$ and $\mathcal{N}$, respectively, where $\mathcal{N}^{*}$ is the set with all feasible assignment patterns, and we set the binary variable, $y_{u, n}$, equal to 0 for all $u \in \mathcal{U}^{*}$ and $n \in \mathcal{N}^{*}$. Next, in lines 2-14 we assign the best assignment pattern to each UE, prioritizing the UEs in worst channel conditions, letting them choose their best (and possibly scarce) RBs in set $\mathcal{N}^{*}$. Thus, in line 3, we find the UE $u^{+}$with lowest average EE given by $\bar{\xi}_{u}=1 / N \sum_{n \in \mathcal{N}^{*}} \xi_{u, n}$. In line 4 , we define $\mathcal{N}^{+}$as the set of available assignment patterns to $\mathrm{UE} u^{+}$, which is given by the intersection between the set of available assignment patterns of the system, $\mathcal{N}^{\dagger}$, and the set of feasible assignment pattern of UE $u^{+}, \mathcal{N}_{u^{+}}^{*}$. Then in lines 5-12, we check which assignment pattern will be allocated to UE $u^{+}$. For that, in line 6 , we select the assignment pattern $n^{+}$with highest EE. Note that, depending on the assignment pattern $n^{+}$assigned to UE $u^{+}$, the number of available RBs can be insufficient to fulfill the data rate requirements of the remaining UEs in set $\mathcal{U}^{+}$. To solve this issue, we assume an approach in which we estimate the minimum number of RBs needed by each UE $u \in \mathcal{U}^{+}$ with $u \neq u^{+}$to fulfill their data rate requirements using the following relation: $\left\lceil\tau_{u} / r_{M}\right\rceil$, where $\lceil\cdot\rceil$ is the ceiling operator. Note that we assume that the UE is using the highest MCS level. Then, we verify if the number of available RBs is higher than the sum of the minimum number of RBs needed to fulfill the data rate requirements of the remaining UEs in set $\mathcal{U}^{+}$(line 7 ). In the negative case, we remove $n^{+}$from set $\mathcal{N}^{+}$in line 10 and repeat the loop until an assignment pattern is assigned to $\mathrm{UE} u^{+}$or the set $\mathcal{N}^{+}$is empty. In the positive case, in line 8 , we

\footnotetext{
${ }^{1}$ An outage event occurs due to the suboptimality of the solutions or when there is no feasible solution even to the optimal solution shown in Section IV.
}

allocate the assignment pattern $n^{+}$to $\mathrm{UE} u^{+}$, remove it from set $\mathcal{N}^{\dagger}$ and we set $\mathcal{N}^{+}$equal to empty. After that, we remove $\mathrm{UE} u^{+}$from set $\mathcal{U}^{+}$and repeat the loop until set $\mathcal{U}^{+}$is empty. Finally, we return the binary variable, $y_{u, n}$, for all $u \in \mathcal{U}^{*}$ and $n \in \mathcal{N}^{*}$ with the found assignment. In addition, the decisions about resource allocation at eNBs should be signaled to the UEs (in downlink direction) for proper uplink transmission.

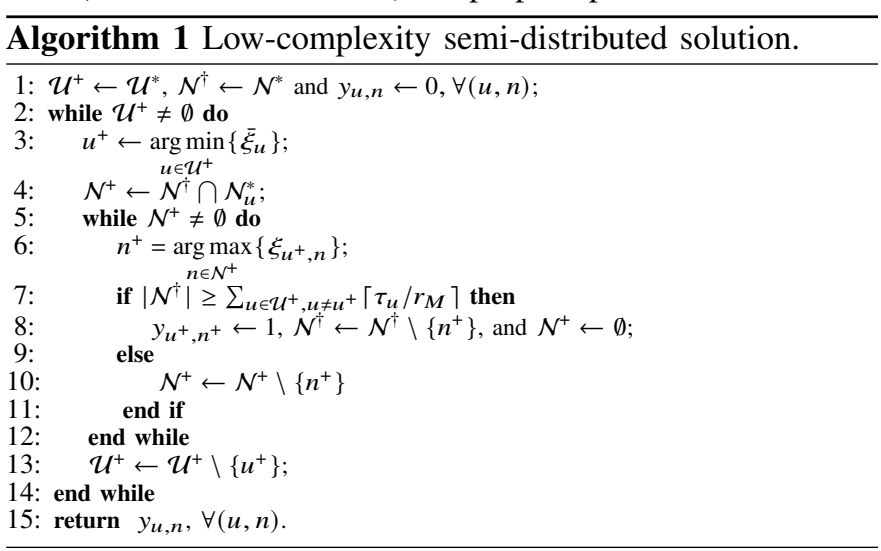

\section{Computational COMPlexity AND Signaling}

As in [8], [9], we consider summations, multiplications and comparisons as the most relevant and time-consuming operations. Therefore, the centralized optimal solutions have exponential worst-case computational complexity given by $O\left(2^{U N M+U}\right)$, see [8] and [9] for more details. Regarding the proposed semi-distributed solution, the most computational complex operations consist in finding the UE in line 3 and the assignment pattern in line 6 . An algorithm that could be used for this task is the MergeSort that according to [12] has worstcase complexity $O(l \log (l))$ where $l$ is the length of the input. Then, we have that the complexity of the semi-distributed solution is, approximately, $O(U(U \log (U)+N \log (N)))$, i.e., it has a polynomial worst-case computational complexity.

Regarding the signaling aspects, we have that each UE $u$ must report $N_{u}^{*}$ scalars with the indices of the feasible assignment patterns plus $N_{u}^{*}$ scalar parameters representing the EE for each assignment pattern, i.e., only $2 N_{u}^{*}$ parameters must be shared between the UE $u$ and the eNB. Such a signaling alleviates the network task of scheduling patterns, since it eliminates part of the optimization tasks usually performed at the network side, i.e., satisfy UE requirements.

\section{PERFormance EVAluation}

We assume the uplink of an LTE network with parameters $\left\{U, K, S, U_{s}\right\}=\{8,15,2,4\}$. The path loss model $^{2}$ is given by $\mathrm{PL}(\mathrm{dB})=35.3+37.6 \log (d)$ and we assume a $\log$-normal shadowing component with standard deviation of $8 \mathrm{~dB}$ and a Rayleigh-distributed fast fading component, as specified in [13]. The link adaptation is realized based on the 15 channel quality indicator (CQI) values used by LTE [14] and the SNR thresholds for switching MCS levels were obtained from [15]. We set $P_{j}^{\max }$ equal to $24 \mathrm{dBm}$ and the circuit power, $\varsigma$, is equal to $0.2 \mathrm{~W}$. Also, the results are obtained from 3,000 different realizations. We assume $\psi_{1}=4$ and $\psi_{2}=3$. We admit that

\footnotetext{
${ }^{2} d$ is a distance between $\mathrm{eNB}$ and UE in meters
} 


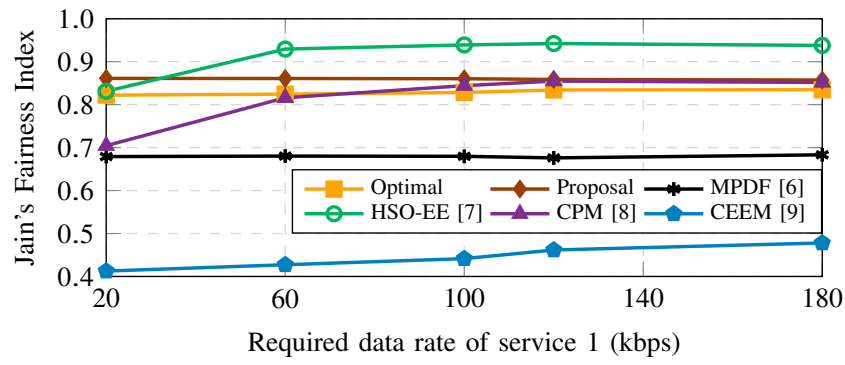

Fig. 1. Jain's fairness index versus required data rate of service 1 .

UEs from service 2 demand a throughput $20 \mathrm{kbps}$ higher than the UEs from service 1 and we assume that the required data rate of UEs from service 1 vary from $20 \mathrm{kbps}$ to $180 \mathrm{kbps}$ with steps of $40 \mathrm{kbps}$. Finally, we compare our solution with: 1) MPDF algorithm from [6]; 2) HSO-EE algorithm from [7]; 3) CPM solution from [8] and; 4) CEEM solution from [9].

In Fig. 1 we show the Jain's fairness index ${ }^{3}$ for all solutions. As we can see, CEEM has the worst performance. This occurs because this solution dedicates more resources to UEs with good channel conditions, which achieve high EE while UEs with worst channel conditions have low EE. On the other hand, the HSO-EE algorithm presents the best performance in terms of Jain's fairness index. The reason is that it allocates only the power necessary to fulfill the data rate requirements of all UEs. The same is valid to CPM, however, to minimize the power consumption it fulfills only the minimum number of UEs in each service, which reduces its fairness. MPDF and the proposed solution present moderate Jain's fairness index. Even so, we note that Jain's fairness index of the proposed solution is higher than 0.8 for all loads.

However, the maximum Jain's fairness index occurs when all EEs are equal, independently of their values. Thus, even when these solutions achieve low EE values, Jain's fairness index can be high. Therefore, in Fig. 2 we present the minimum EE versus the QoS levels. As we can see, the minimum EE decreases as the QoS levels increase for the proposed solutions. This occurs because the power consumption increases in order to fulfill the QoS demand. Another interesting result is that the minimum EE of CPM and CEEM solutions are zero for all simulated loads. The reason is that these solutions fulfill only the minimum number of UEs of each service. Since $\psi_{2}=3$, one of the UEs of service 2 is always discarded and no resource is allocated to it, consequently, its EE is zero. On the other hand, our proposed solutions aim to maximize the minimum EE, thus, they always allocate resource for all UEs whenever possible. Indeed, the proposed solutions discard one of the UEs of service 2 only when this is necessary to avoid outage, i.e., to guarantee that the system requirements are fulfilled. In addition, the proposed solutions outperform the state-of-art algorithms for all loads. The reason for this is that, in general, the other solutions focus on UEs with good channel condition in order to maximize the overall $\mathrm{EE}$ or to minimize the power consumption of the system. On the other hand, the proposed solutions dedicate more resources to the UEs in worst channel conditions in order to maximize the minimum

\footnotetext{
${ }^{3}$ Note that Jain's index is applied in the EE instead of the data rate.
}

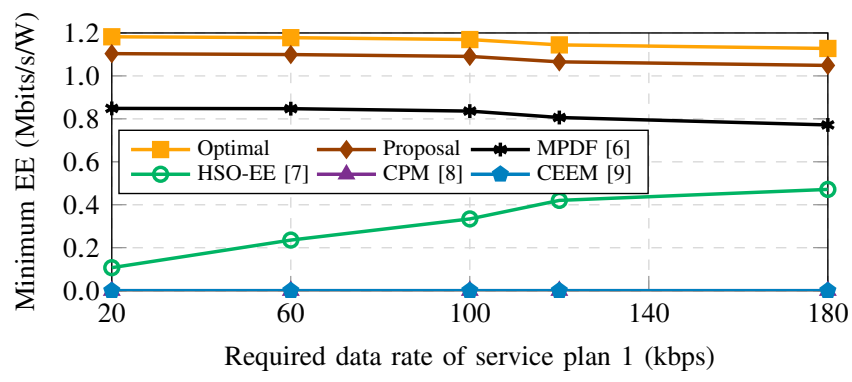

Fig. 2. Minimum EE versus required data rate of service 1.

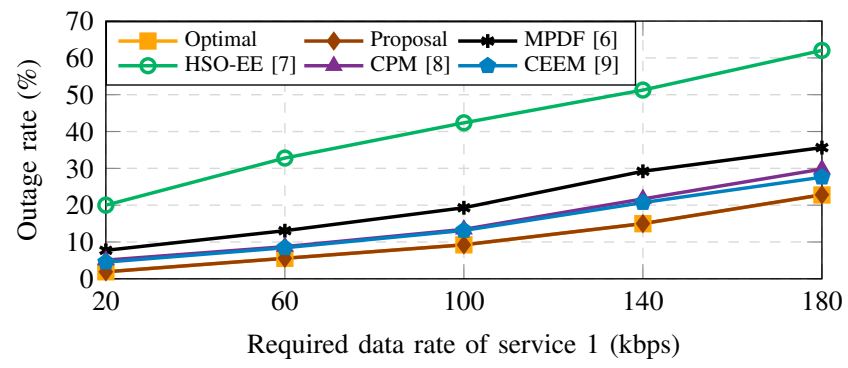

Fig. 3. Outage rate versus required data rate of service 1 .

EE of the system. Also, comparing the performance of the semi-distributed solution to that of the centralized optimal solution, we have that the former performs very close to the centralized optimal solution for all loads.

In Fig. 3 we present the outage rate versus the QoS levels for all algorithms. As expected, the outage rate increases as the QoS levels augment. Furthermore, we observe that MDPF and HSO-EE solutions present the worst performances in term of outage rate. This occurs because these solutions do not consider a minimum number of UEs per service, i.e., all UEs must have their QoS demands fulfilled. The other solutions, in their turn, can select only the three UEs with better channel conditions in service 2, instead of fulfilling the QoS demand of all UEs. Also, we can see that the proposed semi-distributed solution presents the same outage rate of the centralized optimal solution for all simulated loads. This occurs because the UEs inform to eNB which are the feasible assignment patterns of them, then, the eNB needs only to select the better assignment pattern in order to maximize the objective function, which can be done efficiently by Alg. 1 .

\section{CONCLUSIONS}

In this letter, we studied the max-min EE problem considering minimum user satisfaction and adjacency constraints in the uplink of LTE networks. The formulated problem consists in a nonlinear combinatorial optimization problem. By means of mathematical manipulations, we converted it into an ILP and solved it using conventional methods in a centralized way, however, at a high computational cost. Thus, we proposed a low-complexity semi-distributed solution using a specific signaling scheme and through computational simulations, we have shown that it performs closely to the centralized optimal solution and outperforms state-of-art algorithms both in terms of minimum EE and outage rate. As a perspective of this study, we indicate the analysis of the presented problem considering a more general fading model, imperfect CSI and multiple antennas at the eNB and UEs. 


\section{REFERENCES}

[1] 3GPP, "NR; physical layer procedures for data," 3rd Generation Partnership Project (3GPP), Tech. Rep 38.214, Oct. 2020.

[2] H. Yang, F. Ren, C. Lin, and J. Zhang, "Frequency-domain packet scheduling for 3GPP LTE uplink," in Proc. IEEE INFOCOM. San Diego, CA, USA: IEEE, Mar. 2010, pp. 1-9, doi: 10.1109/INFCOM.2010.5462089.

[3] S. Sun and S. Moon, "Practical scheduling algorithms with contiguous resource allocation for next-generation wireless systems," IEEE Wireless Communications Letters, vol. 10, no. 4, pp. 725-729, Apr. 2020, doi: 10.1109/LWC.2020.3041836.

[4] A. Ahmad and N. Shah, "A joint resource optimization and adaptive modulation framework for uplink single-carrier frequency-division multiple access systems," International Journal of Commun. Syst., vol. 28, no. 3, pp. 437-456, Oct. 2015, doi: 10.1002/dac.2677.

[5] D. Triantafyllopoulou, K. Kollias, and K. Moessner, "QoS and energy efficient resource allocation in uplink SC-FDMA systems," IEEE Trans. Wireless Commun., vol. 14, no. 6, pp. 3033-3045, Jun. 2015, doi: 10.1109/TWC.2015.2399916.

[6] Z. Zheng, Q. Ji, L. Dan, and S. Li, "Energy-efficient power allocation for uplink SC-FDMA," in Proc. IEEE International Conference on Wireless Communications, Networking and Mobile Computing. Shanghai, China: IEEE, Sep. 2012, pp. 1-4, doi: 10.1109/WiCOM.2012.6478514.

[7] F. A. Orakzai, A. Ahmad, M. T. R. Khan, and M. Iqbal, "Optimal energy-efficient resource allocation in uplink sc-fdma networks," Trans. Emerging Telecommunications Technol., vol. 28, no. 8, p. e3153, Feb. 2017, doi: 10.1002/ett.3153.

[8] I. M. Braga Jr., F. R. M. Lima, T. F. Maciel, and F. R. P. Cavalcanti, "Transmit power minimization with QoS and user satisfaction guarantees in SC-FDMA systems," in Proc. IEEE Wireless Commun. and Networking Conference (WCNC). Barcelona, Spain: IEEE, Apr. 2018, pp. 1-6, doi: 10.1109/WCNC.2018.8377301.

[9] I. M. Braga Jr, F. R. M. Lima, T. F. Maciel, and F. R. P. Cavalcanti, "Maximizing energy efficiency in SC-FDMA uplink with QoS guarantees and user satisfaction," Trans. Emerging Telecommunications Technol., vol. 30, no. 12, p. e3674, Jul. 2019, doi: 10.1002/ett.3674.

[10] T. F. Rahman and C. Sacchi, "A cooperative radio resource management strategy for mobile multimedia LTE uplink," in IEEE Aerospace Conference. Big Sky, MT, USA: IEEE, Mar. 2014, pp. 1-8, doi: 10.1109/AERO.2014.6836180.

[11] G. L. Nemhauser and L. A. Wolsey, Integer and Combinatorial Optimization. John Wiley \& Sons, 1988.

[12] J. Katajainen, T. Pasanen, and J. Teuhola, "Practical in-place mergesort," Nord. J. Comput., vol. 3, no. 1, pp. 27-40, 1996.

[13] F. R. M. Lima, T. F. Maciel, and F. R. P. Cavalcanti, "Radio resource allocation in SC-FDMA uplink with resource adjacency constraints," Journal of Communication and Information Syst., vol. 31, no. 1, Oct. 2016, doi: 10.14209/jcis.2016.24

[14] 3GPP, "Evolved universal terrestrial radio access (E-UTRA); physical layer procedures," 3rd Generation Partnership Project (3GPP), Tech. Rep 36.213, May 2017.

[15] C. Mehlführer, M. Wrulich, J. C. Ikuno, D. Bosanska, and M. Rupp, "Simulating the long term evolution physical layer," in Proc. IEEE European Signal Processing Conference. Glasgow, UK: IEEE, Apr. 2009, pp. 1471-1478. 